DOE/NV/25946--131

SPIE Optics and Photonics Conference, August 26-30, 2007, San Diego, CA

\title{
Design, construction, alignment, and calibration of a compact velocimetry experiment
}

Morris I. Kaufman, Robert M. Malone, Brent C. Frogget, Vincent T. Romero, David L. Esquibel, Adam Iverson, Gregory A. Lare, Bart Briggs, Douglas DeVore, Brian Cata National Security Technologies, LLC, Los Alamos Operations

Kevin McGillivray, Martin Palagi

National Security Technologies, LLC, North Las Vegas Operations

Matthew E. Briggs, Michael R. Furlanetto, David B. Holtkamp, Nick S.P. King, Mark D. Wilke

Los Alamos National Laboratory

Michael D. Furnish

Sandia National Laboratories

\section{ABSTRACT}

A velocimetry experiment has been designed to measure shock properties for small, cylindrical, metal targets ( $8 \mathrm{~mm}$ diameter $\times 2 \mathrm{~mm}$ thick). A target is accelerated by high explosives, caught, then retrieved for later inspection. The target is expected to move at a velocity of 0.1 to $3 \mathrm{~km} / \mathrm{sec}$. The complete experiment canister is $\sim 105 \mathrm{~mm}$ in diameter and $380 \mathrm{~mm}$ long. Optical velocimetry diagnostics include the Velocity Interferometer System for Any Reflector (VISAR) and photon Doppler velocimetry (PDV). The packaging of the velocity diagnostics is not allowed to interfere with the foam catchment or an X-ray imaging diagnostic. Using commercial lenses, a single optical relay collects Doppler-shifted light for both VISAR and PDV. The use of fiber optics allows measurement of point velocities on the target surface for accelerations lasting for $3 \mathrm{~mm}$ of travel. Operating at $532 \mathrm{~nm}$, the VISAR has separate illumination fibers requiring alignment. The PDV diagnostic operates at $1550 \mathrm{~nm}$ but is aligned and calibrated at $670 \mathrm{~nm}$. VISAR and PDV diagnostics are complimentary measurements that image spots in close proximity on the target surface. Because the optical relay uses commercial glass, optical fibers' axial positions are offset to compensate for chromatic aberrations. The optomechanical design requires careful attention to fiber management, mechanical assembly and disassembly, foam catchment design, and X-ray diagnostic field of view. Calibration and alignment data are archived at each assembly sequence stage. The photon budgets for the VISAR and PDV diagnostics are separately estimated.

Keywords: velocimetry, VISAR, PDV, calibration, alignment, photon budget, fiber optics, optomechanics, fast VISAR, fast PDV

This work was done by National Security Technologies, LLC, under Contract No. DE-AC52-06NA25946 with the U.S. Department of Energy. 